\title{
Impact of the Time Interval Between Primary Debulking Surgery and Start of Adjuvant Chemotherapy in Advanced Epithelial Ovarian Cancer
}

\author{
Hao Lin' \\ Wen-Hsin Chen' \\ Chen-Hsuan Wu ${ }^{1,2}$ \\ Yu-Che Ou ${ }^{3}$ \\ Yu-Jen Chen ${ }^{3}$ \\ Ying-Yi Chen' \\ Yu-Han Lin ${ }^{4}$ \\ Hung-Chun Fu (iD) ${ }^{1-3}$ \\ 'Department of Obstetrics and \\ Gynecology, Kaohsiung Chang Gung \\ Memorial Hospital and Chang Gung \\ University College of Medicine, \\ Kaohsiung, Taiwan; ${ }^{2}$ Graduate Institute of \\ Clinical Medical Sciences, Chang Gung \\ University, Lin-Kou, Taiwan; ${ }^{3}$ Department \\ of Obstetrics and Gynecology, Chia-Yi \\ Chang Gung Memorial Hospital, Chia-Yi, \\ Taiwan; ${ }^{4}$ Department of Obstetrics and \\ Gynecology, National Cheng Kung \\ University Hospital, Tainan, Taiwan
}

\begin{abstract}
Aim: To investigate whether the time interval between primary debulking surgery (PDS) and initiating adjuvant chemotherapy affects survival in patients with epithelial ovarian cancer (EOC).

Methods: We retrospectively reviewed FIGO stage IIB to IV EOC patients who received PDS followed by adjuvant chemotherapy in our hospital between January 2008 and December 2016. The optimal cut-off time interval to chemotherapy related to survival was determined using the Contal and O'Quigley method and Cox hazard models. Cox regression analysis was used to identify the independent effect of time interval on survival.

Results: A total of 152 patients were identified and divided into three groups based on the time interval between PDS and initiating adjuvant chemotherapy: early ( $<23$ days), intermediate (23-43 days) and late ( $>43$ days). The intermediate group had a significantly better median progression-free survival (PFS, 35.5 months) compared to the early (20 months) and late (22.6 months) groups. After adjustments for confounding factors, time interval was still an independent variable affecting PFS. The intermediate group was associated with a better PFS compared with the early and late groups (hazard ratio 0.27 , 95\% CI $0.10-0.83$, $p=0.002)$. There was no statistical significance in overall survival (OS) in univariate or multivariate analysis, although there was a trend towards better OS in the intermediate group. Conclusion: Our results provide evidence that the time interval from PDS to chemotherapy influences PFS in patients with advanced EOC. The optimal time to initiate chemotherapy was between 23 and 43 days, within 3-6 weeks post-operatively. Initiating chemotherapy early $(<23$ days) did not appear to benefit PFS.
\end{abstract}

Keywords: time interval, chemotherapy, ovarian cancer

\section{Introduction}

Ovarian cancer is the seventh common cancers of women and the third common gynecologic malignancy worldwide. ${ }^{1}$ It is the most leading cause of deaths from gynecologic cancers. International Federation of Obstetrics and Gynecology (FIGO) stage, tumor grade, histological subtype and residual tumor after surgery are the established prognostic factors of progression-free survival (PFS) and overall survival (OS) for women with epithelial ovarian cancer (EOC). ${ }^{2}$ The standard treatment for advanced-stage EOC is primary debulking surgery (PDS) followed by adjuvant chemotherapy. ${ }^{3,4}$ Adjuvant chemotherapy is important to eliminate
Correspondence: Hung-Chun Fu Department of Obstetrics and Gynecology, Kaohsiung Chang Gung Memorial Hospital, I23, Ta Pei Road, Niao Sung District, Kaohsiung City, Taiwan, Republic of China $\mathrm{Tel}+8867731712$ Fax +88677322915

Email allen133@adm.cgmh.org.tw 
residual diseases or microscopic diseases. Critical factors related to primary chemotherapy include inherent and acquired drugs resistance, and the timing of initiation of chemotherapy following PDS. ${ }^{5}$

Some previous studies showed that early initiation of chemotherapy following surgery was more benefit for ovarian cancer patients' survivals. ${ }^{5}$ According to the Gompertzian model, the tumor burden is lower after debulking surgery and cells will divide more rapidly due to the effect of cytokines, and this rapid cell cycle is then a good target for chemotherapeutic action. ${ }^{6}$ Therefore, it is generally accepted that adjuvant chemotherapy should not be delayed after debulking surgery. However, initiating chemotherapy too quickly after major surgery may lead to more adverse effects and possibly interrupt the following treatment plan. Over the past decades, several studies have investigated the best time to initiate chemotherapy after completing surgery to delay disease recurrence in a wide variety of malignancies. ${ }^{5,72}$ The optimal time to start chemotherapy after surgery in patients with EOC has yet to be well established. Therefore, in this study, we aimed to investigate whether different time intervals between PDS and initiating adjuvant chemotherapy affect the survival of patients with advanced EOC.

\section{Materials and Methods}

We retrospectively reviewed patients with advanced EOC from FIGO (International Federation of Gynecology and Obstetrics) stage IIB to IV who received PDS followed by adjuvant chemotherapy in our hospital between January 2008 and December 2016. Patients were excluded if they underwent interval debulking surgery after neoadjuvant chemotherapy, had received less than three courses of adjuvant chemotherapy in stage IIB and six courses in stage III/IV, or did not receive follow-up. Data acquired from medical records included age, pretreatment cancer antigen 125 (CA-125) level, FIGO stage, ascites amount, histologic type and grade, surgical debulking extent, residual tumor, chemotherapy regimen, and the use of bevacizumab in front-line treatment. The time interval to chemotherapy was defined as the period between the date of surgery and the first date of initiating chemotherapy. After completing treatment, all patients were followed up every 3 months in the first 2 years, every 3-6 months in the following 3 years, and then every 6-12 months thereafter. Details of clinical symptoms and pelvic examinations were recorded, and tumor marker, chest X-ray, and computed tomography (if necessary) examinations were arranged. The Institutional Review Board of Chang Gung Memorial Hospital approved this study (number: 201601487B0C501).

The optimal cut-off time interval for chemotherapy related to survival was determined using the Contal and O'Quigley method and Cox hazard models. The patients were then categorized into different groups according to the time of initiating adjuvant chemotherapy after surgery. Differences between proportions were evaluated using the chi-square test. Univariate Cox regression analysis was performed first with each clinicopathological factor including time to chemotherapy. The variables reaching statistical significance were then entered into multivariate Cox proportional hazards analysis to identify the most significant independent prognostic factors for PFS and OS. To evaluate the efficacy of adjuvant chemotherapy according to the timing of its initiation, we analyzed and compared the 5-year PFS and OS rates after surgery among the groups. The PFS and OS curves were estimated using the Kaplan-Meier method, and differences between groups were assessed using the Log rank test. A $P$-value of less than 0.05 was considered to represent statistical significance. All statistical analyses were performed using SPSS version 20.0 (IBM Co., Armonk, NY, USA).

\section{Results}

A total of 152 eligible patients were identified for analysis, and their baseline characteristics are listed in Table 1. The median time from surgery to the initiation of adjuvant chemotherapy was 29 days (interquartile range 24-37 days). The best cut-off points of the time interval to chemotherapy based on Contal and O'Quigley's method and Cox hazard model were 23 and 43 days (Figure 1). Therefore, we categorized the patients into three groups according to the time interval to chemotherapy: early group ( $<23$ days), intermediate group (23-43 days), and late group ( $>43$ days). There were no significant differences in terms of age, FIGO stage, histology, tumor grade, Eastern Cooperative Oncology Group (ECOG) performance status, ascites amount, chemotherapy regimen, and the use of front-line bevacizumab among the groups. However, intermediate group had more optimal debulking surgery, while the late group had a significantly higher pretreatment CA-125 level, more extensive surgery (defined as received at least one of the following procedures including intestine resection, hepatectomy, splenectomy, partial diaphragmectomy, cystectomy, or nephrectomy), and longer intensive care unit (ICU) stay. 
Table I Characteristics and Demographics of the Patients $(\mathrm{N}=152)$

\begin{tabular}{|c|c|}
\hline Characteristics & Number (\%) \\
\hline $\begin{array}{l}\text { Age at diagnosis, median (inter-quartile range), } \\
\text { years }\end{array}$ & $54.5(46.5-61)$ \\
\hline $\begin{array}{l}\text { Time to chemotherapy, median (interquartile } \\
\text { range), days }\end{array}$ & $29(24-37)$ \\
\hline FIGO stage, n (\%) & \\
\hline IIB & $30(19.7)$ \\
\hline III & $106(69.7)$ \\
\hline IV & $16(10.5)$ \\
\hline Histology, n (\%) & \\
\hline High-grade serous & $73(48.0)$ \\
\hline Low-grade serous & $8(5.3)$ \\
\hline Clear cell & $27(17.8)$ \\
\hline Endometrioid & $19(12.5)$ \\
\hline Others $^{\dagger}$ & $25(16.4)$ \\
\hline Grade*, n (\%) & \\
\hline Grade I & $13(8.6)$ \\
\hline Non-Grade I & $139(91.4)$ \\
\hline ECOG performance status, n (\%) & \\
\hline 0 & $143(94.0)$ \\
\hline I & $6(3.9)$ \\
\hline 2 & $3(2.1)$ \\
\hline CA-I25 at diagnosis, $n(\%)$ & \\
\hline$<400 \mathrm{U} / \mathrm{mL}$ & $60(39.4)$ \\
\hline$\geq 400 \mathrm{U} / \mathrm{mL}$ & $92(60.6)$ \\
\hline Extensive surgery ${ }^{\ddagger}, \mathrm{n}(\%)$ & \\
\hline Yes & $40(26.3)$ \\
\hline No & $112(73.7)$ \\
\hline Postoperative residual tumor, $\mathrm{n}(\%)$ & \\
\hline$\leq \mathrm{lcm}$ (optimal) & $113(74.3)$ \\
\hline >I cm (suboptimal) & $39(25.7)$ \\
\hline Ascites, n (\%) & \\
\hline$<500 \mathrm{~mL}$ & $84(55.4)$ \\
\hline$\geq 500 \mathrm{~mL}$ & $68(44.6)$ \\
\hline Chemotherapy regimen, n (\%) & \\
\hline Carboplatin + Paclitaxel & 139 (91.4) \\
\hline Others & $13(8.6)$ \\
\hline Intensive care unit stay, n (\%) & \\
\hline Yes & I7 (I I.2) \\
\hline No & I 35 (88.8) \\
\hline
\end{tabular}

Notes: *Non-GI including grade 2 , grade 3 , undifferentiated, and clear cell carcinoma; ${ }^{\dagger}$ Including mucinous, transitional, undifferentiated, mixed type; ${ }^{\ddagger}$ Including intestine resection, hepatectomy, splenectomy, partial diaphragmectomy, cystectomy, or nephrectomy.

Abbreviations: ECOG, Eastern Cooperative Oncology Group; FIGO, International Federation of Obstetrics and Gynecology.
Detailed baseline characteristics of the three groups are shown in Table 2.

The 5-year PFS rates were $30 \%$ in the early group, $68 \%$ in the intermediate group, and $10 \%$ in the late group (Log rank, $p<0.05$ ) (Figure 2), while the 5-year OS rates were $57 \%$ in the early group, $72 \%$ in the intermediate group, and $30 \%$ in the late group (Log rank, early vs intermediate $p=0.119$; intermediate vs late $p=0.091$ ) (Figure 3). The intermediate group had a significantly better median PFS (35.5 months) compared to the early and late groups (20 and 22.6 months, respectively). After adjustments for confounding factors, time interval was still an independent variable affecting PFS. The intermediate group was associated with a better PFS compared to the early and late groups (HR 0.41, 95\% CI 0.21-0.81, $p=0.011)$. However, there was no statistical significance in OS in either univariate or multivariate analysis, although there was a trend towards better OS in the intermediate group. Cox proportional hazard regression models for 5-year PFS and OS are shown in Tables 3 and 4.

\section{Discussion}

Our results provide evidence that the time interval from PDS to chemotherapy influences PFS in patients with advanced EOC. The optimal time to initiate chemotherapy was between 23 and 43 days, within 3-6 weeks postoperatively. Initiating chemotherapy $<23$ and $>43$ days did not appear to benefit PFS. Although our data only showed a trend towards better OS in the intermediate group, most patients now receive multiple postprogression treatments, including surgery, chemotherapy and biological-targeted therapies, which can significantly confound and dilute the effects of the time interval to chemotherapy on the OS endpoint. Therefore, the consensus of the Gynecological Cancer InterGroup (GCIG) concluded that while OS remains the gold standard for demonstrating benefits in clinical trials, PFS is still a relevant primary endpoint for clinical trials of first-line therapies for ovarian cancer. ${ }^{13}$

Studies on whether the time interval between PDS and initiating chemotherapy affects the survival of patients with EOC have been inconclusive. Some studies have suggested that delayed chemotherapy after PDS was not associated with poor survival, while others have reported that the time interval to chemotherapy was an important predictor of survival. The most recent negative result was 


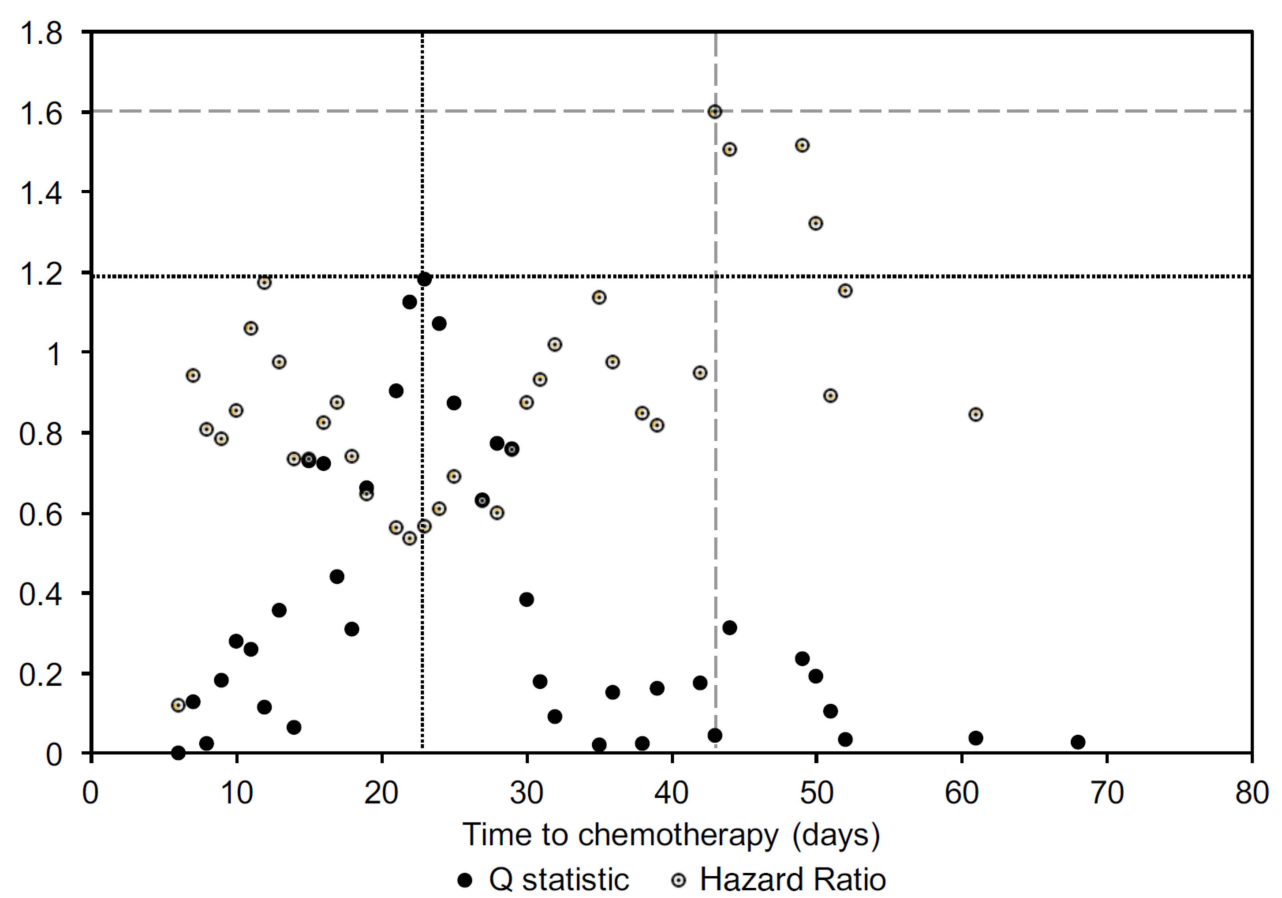

Figure I The best cut-off points of time interval to chemotherapy. The best cut-off points of time interval to chemotherapy based on the analytic results of Contal and O'Quigley's method and Cox hazard models were 23 and 43 days.

from a Danish nationwide population-based study reported by Lydiksen et al, in which they included patients with all stages and found a non-significant increased risk of death with a time interval $>32$ days compared with $\leq 32$ days. ${ }^{14}$ In earlier studies, Aletti et al and Gadducci et al focused on stage IIIC/IV and II-IV patients, respectively, with the time to chemotherapy categorized by quartiles, and they both demonstrated a negative impact of time interval to chemotherapy on survival. ${ }^{9,10}$ In contrast, Timmermans et al and Wright et al demonstrated worse survival when patients started chemotherapy after 37 and 84 days, respectively. ${ }^{15,16}$ Seagle et al reported the most powerful positive result. ${ }^{17}$ They collected data from the National Cancer Data Base and analyzed 15,752 patients representing the overall American population with ovarian cancer, and found that a delay in chemotherapy $>35$ days was associated with a 7\% increased hazard of death. Interestingly, they also found that initiating chemotherapy early ( $<21$ days) was associated with decreased survival, and our data seemed to agree with this finding. ${ }^{17}$ This paradoxical finding is probable due to impaired host defense mechanisms following major surgery leading to intolerance to chemotherapy, which in turn delayed subsequent cycles or led to a reduction in the dosage which thereby mediated the increased hazard of death.
There are several possible explanations for the conflicting results in the optimal timing of chemotherapy in the postoperative period. First, differences in the definition of delayed chemotherapy with regards to the cut-off points. Most studies used median values while some used interquartile range. These differences indicate the heterogeneity of the studied populations and may explain the inconsistent outcomes. Dichotomization of a continuous variable with a median value can result in information loss, and then loss of power to detect actual significance and can sometimes lead to biased estimates in regression settings. ${ }^{18}$ In addition, not all continuous variables have a single cut-off point. Even if a single cut-off point exists and it is statistically significant, one also needs to consider the clinical relevance of such a cut-off point. ${ }^{19}$ In the present study, we did not use median values as the cutoff point, and instead we identified cut-off points based on outcome oriented (time to event) Contal and O'Quigley's method, which may have resulted in cut-off points that were most significantly associated with outcomes.

Second, the evolution of surgical management for ovarian cancer. With advances in surgical techniques and postoperative care, more aggressive cytoreductive surgery to no gross residual tumor is the main goal in current clinical practice. ${ }^{20}$ Previous studies have reported that more extensive cytoreduction increases the occurrence of 
Table 2 Eligible Patient Demographics and Clinical Characteristics According to the Time to Chemotherapy from Primary Debulking Surgery

\begin{tabular}{|c|c|c|c|c|}
\hline \multirow[t]{2}{*}{ Characteristics } & \multicolumn{3}{|c|}{ Time to Chemotherapy (Days) } & \multirow[b]{2}{*}{$P$ value } \\
\hline & $<23$ & $23-43$ & $>43$ & \\
\hline Number of patients & 107 & 32 & 13 & \\
\hline Age at diagnosis, (years) & 54 & 54.5 & 55 & \multirow[t]{2}{*}{0.930} \\
\hline Median (interquartile range) & $(47-61)$ & $(46.5-6 I)$ & $(44-7 I .5)$ & \\
\hline FIGO stage, n (\%) & & & & 0.431 \\
\hline IIB & $19(17.8)$ & $9(28.1)$ & $2(15.4)$ & \\
\hline III & $74(69.2)$ & $22(68.8)$ & $10(76.9)$ & \\
\hline IV & $14(13.1)$ & I (3.I) & I (7.7) & \\
\hline Histology, n (\%) & & & & 0.296 \\
\hline High-grade serous & $57(53.8)$ & II (34.4) & $5(35.8)$ & \\
\hline Low-grade serous & $4(3.8)$ & $3(9.4)$ & I (7.I) & \\
\hline Clear cell & $19(17.8)$ & $7(21.9)$ & I (7.7) & \\
\hline Endometrioid & II (10.3) & $4(12.5)$ & $4(30.8)$ & \\
\hline Others & $15(14.0)$ & $7(21.9)$ & $3(23.1)$ & \\
\hline Grade, n (\%) & & & & 0.391 \\
\hline Grade I & $7(8.0)$ & $4(14.3)$ & $2(16.7)$ & \\
\hline Non-Grade I & $100(92.0)$ & $28(85.7)$ & II (83.3) & \\
\hline ECOG performance status, n (\%) & & & & 0.926 \\
\hline 0 & $100(93.5)$ & $30(93.8)$ & $13(100)$ & \\
\hline $\mathrm{I}$ & $5(4.7)$ & I (3.I) & $0(0.0)$ & \\
\hline 2 & $2(1.9)$ & $\mathrm{I}(3.1)$ & $0(0.0)$ & \\
\hline CA-125 at diagnosis, $n$ (\%) & & & & 0.036 \\
\hline$<400 \mathrm{U} / \mathrm{mL}$ & $37(34.7)$ & $16(61.5)$ & $4(30.8)$ & \\
\hline$\geq 400 \mathrm{U} / \mathrm{mL}$ & $70(65.3)$ & $10(38.5)$ & $9(69.2)$ & \\
\hline Extensive surgery, $\mathrm{n}(\%)$ & & & & $0.026 *$ \\
\hline Yes & $24(22.4)$ & $9(28.1)$ & $7(53.9)$ & \\
\hline No & $83(77.5)$ & $23(71.9)$ & $6(46.2)$ & \\
\hline Postoperative residual tumor, $\mathrm{n}(\%)$ & & & & $0.045^{*}$ \\
\hline$\leq \mathrm{cm}$ (optimal) & $76(71.0)$ & $29(90.6)$ & $8(61.5)$ & \\
\hline >I cm (suboptimal) & $31(29.0)$ & $3(9.4)$ & $5(38.5)$ & \\
\hline Ascites, n (\%) & & & & 0.173 \\
\hline$<500 \mathrm{~mL}$ & $54(50.5)$ & $22(68.8)$ & $8(61.5)$ & \\
\hline$\geq 500 \mathrm{~mL}$ & $53(49.5)$ & $10(31.3)$ & $5(38.5)$ & \\
\hline Chemotherapy regimen, $\mathrm{n}(\%)$ & & & & 0.894 \\
\hline Carboplatin + Paclitaxel & $97(90.7)$ & $30(93.8)$ & $12(92.3)$ & \\
\hline Others & $10(9.3)$ & $2(6.3)$ & I (7.7) & \\
\hline Bevacizumab first-line, n (\%) & & & & 0.083 \\
\hline Yes & II (I0.3) & $0(0.0)$ & $0(0.0)$ & \\
\hline No & $96(89.7)$ & $32(100)$ & $13(100)$ & \\
\hline Intensive care unit stay, n (\%) & & & & $0.009 *$ \\
\hline Yes & $7(6.5)$ & $6(18.8)$ & $4(30.8)$ & \\
\hline No & $100(93.5)$ & $26(81.3)$ & $9(69.2)$ & \\
\hline
\end{tabular}

Note: $* p<0.05$

Abbreviations: ECOG, Eastern Cooperative Oncology Group; FIGO, International Federation of Obstetrics and Gynecology. 


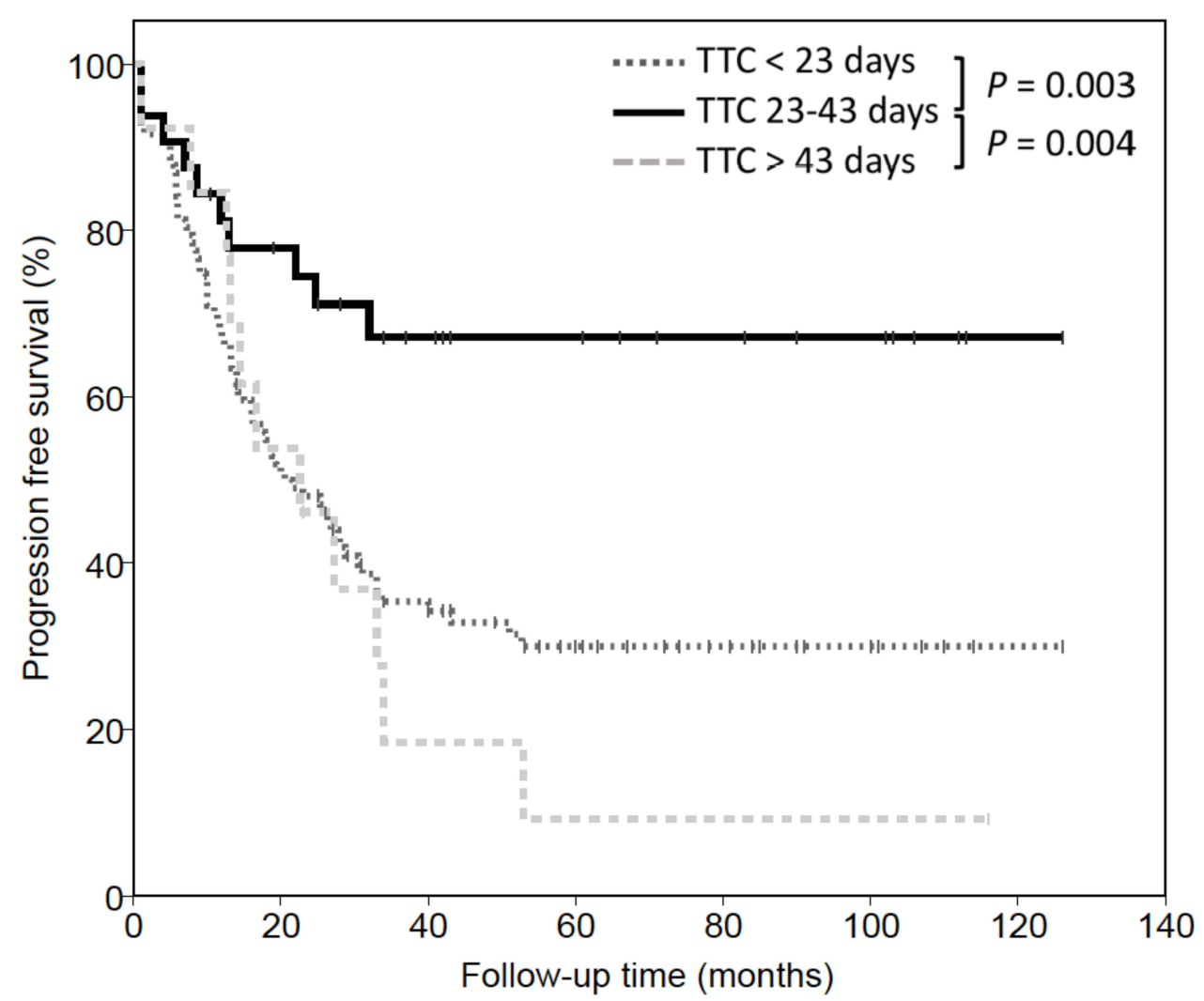

Figure 2 Kaplan-Meier curves for progression-free survival in subgroups of patients stratified by time to chemotherapy (TTC). The 5-year progression-free survival rates were $30 \%$ in the early group, $68 \%$ in the intermediate group, and $10 \%$ in the late group (Log rank, $p<0.05$ ).

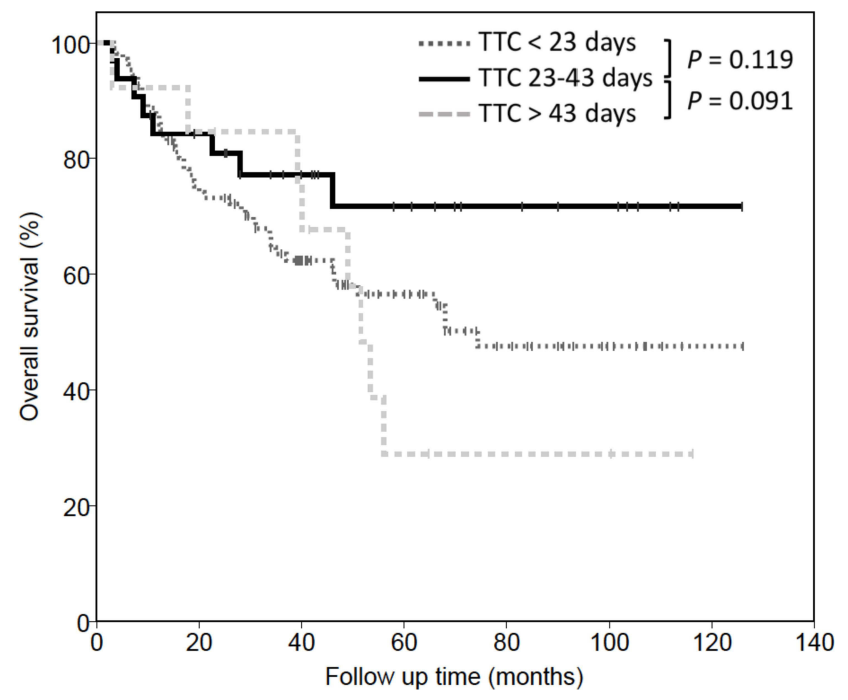

Figure 3 Kaplan-Meier curves for overall survival in subgroups of patients stratified by time to chemotherapy (TTC). The 5 -year overall survival rates were $57 \%$ in the early group, $72 \%$ in the intermediate group, and $30 \%$ in the late group (Log rank, early vs intermediate $p=0.119$; intermediate vs late $p=0.091$ ).

postoperative complications and chemotherapy delay. ${ }^{16,21,22}$ We did find a significantly higher rate of extensive surgery in the late ( $>43$ days) group, but this does not necessarily reflect a higher rate of complete cytoreduction. Fotopoulou et al reported a major complication rate of $18.6 \%$ and a mortality rate of $3.4 \%$ (within 3 months) after extensive cytoreductive surgery for disseminated (FIGO stage IIIC and IV) ovarian cancer. $^{23}$ Although chemotherapy was delayed in only 3.3\% of the patients, the cut-off time they used to define a delay in chemotherapy was 56 days. Furthermore, their median time between surgery and first cycle of postoperative chemotherapy was 37 days, which is longer than in most previous studies ranging from 20 to 40 days, and also ours. ${ }^{9,14,15,24,25}$ Although no gross residual tumor is a well-established good prognostic factor, delayed chemotherapy initiation may attenuate the potential benefit of extensive cytoreductive surgery. Whether the prognosis of patients with no residual disease after extensive surgery followed by delayed chemotherapy is superior to those with macroscopic residual disease and early chemotherapy is still unknown.

Third, the rates of residual disease status after debulking surgery in different studies have been inconsistent, and this may also confound the effect of time to chemotherapy. 
Table 3 Univariate and Multivariate Analyses of Factors Associated with Progression-Free Survival

\begin{tabular}{|c|c|c|c|c|}
\hline \multirow[t]{2}{*}{ Characteristics } & \multirow{2}{*}{$\begin{array}{c}\text { Univariate } \\
\text { HR (95\% Cl) }\end{array}$} & \multirow[b]{2}{*}{$p$-value } & \multirow{2}{*}{$\begin{array}{l}\text { Multivariate } \\
\text { HR }(95 \% \mathrm{Cl})\end{array}$} & \multirow[b]{2}{*}{$p$-value } \\
\hline & & & & \\
\hline \multicolumn{5}{|l|}{ FIGO stage } \\
\hline IIB & I & & I & \\
\hline III & $2.27(1.20-4.30)$ & $0.011 *$ & $2.21(1.05-4.63)$ & $0.035^{*}$ \\
\hline IV & $3.23(1.42-7.34)$ & $0.005^{*}$ & $2.59(0.92-7.24)$ & 0.069 \\
\hline \multicolumn{5}{|l|}{ Histology } \\
\hline High-grade serous & 1 & & & \\
\hline Low-grade serous & $0.49(0.15-1.57)$ & 0.231 & & \\
\hline Clear cell & $1.07(0.6 \mathrm{I}-\mathrm{I} .85)$ & 0.822 & & \\
\hline Endometrioid & $0.49(0.23-1.04)$ & 0.064 & & \\
\hline Others & $1.17(0.65-2.10)$ & 0.583 & & \\
\hline \multicolumn{5}{|l|}{ Grade } \\
\hline Grade I & I & & & \\
\hline Non-Grade I & $2.70(0.98-7.35)$ & 0.053 & & \\
\hline \multicolumn{5}{|l|}{ CA-I25 at diagnosis } \\
\hline$<400 \mathrm{U} / \mathrm{mL}$ & I & & & \\
\hline$\geq 400 \mathrm{U} / \mathrm{mL}$ & $2.10(0.76-1.89)$ & 0.415 & & \\
\hline \multicolumn{5}{|l|}{ Extensive surgery } \\
\hline Yes & 1 & & I & \\
\hline No & $0.49(0.32-0.76)$ & $0.001 *$ & $0.61(0.37-1.02)$ & 0.060 \\
\hline \multicolumn{5}{|l|}{ Residual tumor } \\
\hline$\leq \mathrm{l} \mathrm{cm}$ (optimal) & I & & I & \\
\hline$>$ I cm (suboptimal) & $2.27(1.47-3.50)$ & $<0.00 I^{*}$ & $1.42(0.84-2.39)$ & 0.190 \\
\hline \multicolumn{5}{|l|}{ Ascites } \\
\hline$<500 \mathrm{~mL}$ & 1 & & 1 & \\
\hline$\geq 500 \mathrm{~mL}$ & $2.25(1.47-3.44)$ & $<0.00 I^{*}$ & $1.74(1.11-2.72)$ & $0.014 *$ \\
\hline \multicolumn{5}{|l|}{ Chemotherapy regimen } \\
\hline Carboplatin + Paclitaxel & I & & & \\
\hline Others & $1.57(0.8 \mathrm{I}-3.03)$ & 0.178 & & \\
\hline \multicolumn{5}{|l|}{ Intensive care unit stay } \\
\hline Yes & I & & & \\
\hline No & $0.57(0.32-1.02)$ & 0.058 & & \\
\hline \multicolumn{5}{|l|}{ Bevacizumab treatment } \\
\hline Yes & I & & & \\
\hline No & $1.45(0.70-3.01)$ & 0.312 & & \\
\hline \multicolumn{5}{|l|}{ Time to chemotherapy, days } \\
\hline$<23$ & 1 & & I & \\
\hline $23-43$ & $0.37(0.19-0.720)$ & $0.004 *$ & $0.4 \mathrm{I}(0.2 \mathrm{I}-0.8 \mathrm{I})$ & $0.011 *$ \\
\hline$>43$ & $1.24(0.65-2.34)$ & 0.504 & $0.95(0.46-1.97)$ & 0.899 \\
\hline
\end{tabular}

Note: $* p$ value $<0.05$

Abbreviations: $\mathrm{Cl}$, confidence interval; FIGO, International Federation of Obstetrics and Gynecology; HR, hazard ratio.

There was a higher tendency for clinicians to start chemotherapy as soon as possible in patients with residual macroscopic disease like ours. In contrast, patients with no residual disease were more prone to have a delay in the initiation of chemotherapy, because they required more extensive surgery and had more postoperative 
Table 4 Univariate and Multivariate Analyses of Factors Associated with Overall Survival

\begin{tabular}{|c|c|c|c|c|}
\hline \multirow[t]{2}{*}{ Characteristics } & Univariate & \multirow[b]{2}{*}{$p$-value } & Multivariate & \multirow[b]{2}{*}{ p-value } \\
\hline & HR (95\% Cl) & & HR $(95 \% \mathrm{Cl})$ & \\
\hline \multicolumn{5}{|l|}{ FIGO stage } \\
\hline IIB & 1 & & & \\
\hline III & $2.16(0.97-4.79)$ & 0.057 & & \\
\hline IV & $2.49(0.90-6.89)$ & 0.077 & & \\
\hline \multicolumn{5}{|l|}{ Histology } \\
\hline High-grade serous & I & & & \\
\hline Low-grade serous & $0.56(0.13-2.38)$ & $0.44 I$ & & \\
\hline Clear cell & $1.54(0.8 \mathrm{I}-2.92)$ & 0.182 & & \\
\hline Endometrioid & $0.56(0.22-1.47)$ & 0.244 & & \\
\hline Others & $1.54(0.78-3.03)$ & 0.205 & & \\
\hline \multicolumn{5}{|l|}{ Grade } \\
\hline Grade I & I & & & \\
\hline Non-Grade I & $2.31(0.72-7.38)$ & 0.157 & & \\
\hline \multicolumn{5}{|l|}{ CA-125 at diagnosis } \\
\hline$<400 \mathrm{U} / \mathrm{mL}$ & 1 & & & \\
\hline$\geq 400 \mathrm{U} / \mathrm{mL}$ & $1.03(0.60-1.78)$ & 0.889 & & \\
\hline \multicolumn{5}{|l|}{ Extensive surgery } \\
\hline Yes & 1 & & I & \\
\hline No & $0.58(0.34-0.98)$ & $0.043^{*}$ & $0.75(0.43-1.32)$ & 0.326 \\
\hline \multicolumn{5}{|l|}{ Residual tumor } \\
\hline$\leq \mathrm{l} \mathrm{cm}$ (optimal) & I & & I & \\
\hline$>$ I cm (suboptimal) & $1.95(1.15-3.28)$ & $0.012 *$ & I.52 (0.86-2.7I) & 0.146 \\
\hline \multicolumn{5}{|l|}{ Ascites } \\
\hline$<500 \mathrm{~mL}$ & I & & I & \\
\hline$\geq 500 \mathrm{~mL}$ & $1.95(1.16-3.29)$ & $0.012^{*}$ & $1.70(0.98-2.92)$ & 0.056 \\
\hline \multicolumn{5}{|l|}{ Chemotherapy regimen } \\
\hline Carboplatin + Paclitaxel & I & & & \\
\hline Others & $1.15(0.49-2.69)$ & 0.732 & & \\
\hline \multicolumn{5}{|l|}{ Intensive care unit stay } \\
\hline Yes & I & & & \\
\hline No & $0.57(0.29-1.12)$ & 0.107 & & \\
\hline \multicolumn{5}{|l|}{ Bevacizumab treatment } \\
\hline Yes & I & & & \\
\hline No & $1.22(0.38-3.94)$ & 0.732 & & \\
\hline \multicolumn{5}{|l|}{ Time to chemotherapy, days } \\
\hline$<23$ & I & & & \\
\hline $23-43$ & $0.54(0.25-1.16)$ & 0.116 & & \\
\hline$>43$ & $1.26(0.59-2.68)$ & 0.537 & & \\
\hline
\end{tabular}

Note: *p value $<0.05$

Abbreviations: $\mathrm{Cl}$, confidence interval; FIGO, International Federation of Obstetrics and Gynecology; HR, hazard ratio.

complications. ${ }^{10}$ Some studies have performed subgroup analysis focusing on residual disease status; however the results have been inconsistent. Hofstetter et al and Tewari et al reported that the delayed initiation of chemotherapy may compromise OS in patients with advanced serous ovarian cancer only when debulking is suboptimal. ${ }^{5,21}$ 
This seems rational considering that residual masses are believed to be more sensitive to chemotherapy soon after debulking surgery. Thus, the timing of chemotherapy may be more relevant in patients with suboptimal debulking surgeries. However, Timmermans et al and Mahner et al found that delayed chemotherapy may decrease survival only in patients with no gross residual disease. ${ }^{16,26}$ They hypothesized that microscopic residual disease may be forced into an accelerated proliferation phase after complete debulking, as suggested by mouse models. ${ }^{27}$ Furthermore, the loss of ovarian function soon after surgery may induce tumor angiogenesis leading to the rapid growth of microscopic residual tumor cells in ovarian cancer patients. ${ }^{28}$

Incorporation of bevacizumab has been accepted as a standard of care in the primary treatment of advanced EOC since the results of ICON7 and GOG-218. . $^{29,30}$ According to these Phase III trials, the PFS was in favor of bevacizumab group; however, OS benefit was only observed in poor-prognosis patients, such as suboptimal debulking status or FIGO stage IV disease. In our clinical practice, we start to use this drug since 2014 and therefore only 11 patients in our study received front-line bevacizumab treatment combining with chemotherapy. Of the 11 patients, all were in the early group and 7 of them had suboptimal debulking status. No PFS and OS benefits were observed in these patients. The unexpected results may be caused by a lack of consistency in maintenance treatment cycle. In our entire cohort, we found a borderline significant of higher optimal debulking rate in the intermediate group. It is generally believed that better PFS in intermediate group might be due to residual tumor status rather than time to chemotherapy but why the significance of this important factor disappeared upon multivariate analysis. We speculate that the bevacizumab application may be considered as a confounding factor and neutralized the effect of residual tumor. Further study evaluating the association between time to chemotherapy and survival should include more patients with the use of standardized bevacizumab treatment.

There are several limitations to this study. First, this was a retrospective study with a single-center design, and post-progression management of patients was different across the study period. Second, there was selection bias since some patients were excluded owing to incomplete staging surgery, inadequate chemotherapy, or being lost to follow-up, although we assumed that these missing patients were at random. Third, we cannot differentiate cases between complete resection and residual tumor
$<1 \mathrm{~cm}$ in optimal debulking group due to unclear medical chart records and therefore subgroup analysis focusing on different residual disease status was impossible.

\section{Conclusions}

We provide evidence that the time interval from PDS to chemotherapy influences survival in patients with advanced EOC. The optimal time to initiate chemotherapy was between 23 and 43 days, within 3-6 weeks postoperatively. Initiating chemotherapy early ( $<23$ days) did not appear to benefit survival. Further nationwide population-based studies with more consistent cohorts are warranted to better elucidate the best timing of initiating chemotherapy after debulking surgery.

\section{Acknowledgments}

This work was supported by a research grant from Chang Gung Memorial Hospital [CMRPG8G0111-3, CMRPG8F1221 and CMRPG8J1311]. We also thank the Biostatistics Center of Kaohsiung Chang Gung Memorial Hospital for assistance with the statistical analysis.

\section{Disclosure}

The authors report no conflicts of interest in this work.

\section{References}

1. Sung H, Ferlay J, Siegel RL, et al. Global cancer statistics 2020: GLOBOCAN estimates of incidence and mortality worldwide for 36 cancers in 185 countries. CA Cancer J Clin. 2021;71(3):209-249. doi: $10.3322 /$ caac. 21660

2. Gourley C, Bookman MA. Evolving concepts in the management of newly diagnosed epithelial ovarian cancer. J Clin Oncol. 2019;37 (27):2386-2397. doi:10.1200/JCO.19.00337

3. Armstrong DK, Alvarez RD, Bakkum-Gamez JN, et al. Ovarian cancer, version 2.2020, NCCN clinical practice guidelines in oncology. J Natl Compr Canc Netw. 2021;19(2):191-226. doi:10.6004/ jnccn.2021.0007

4. Kuroki L, Guntupalli SR. Treatment of epithelial ovarian cancer. BMJ. 2020;371:m3773. doi:10.1136/bmj.m3773

5. Tewari KS, Java JJ, Eskander RN, Monk BJ, Burger RA. Early initiation of chemotherapy following complete resection of advanced ovarian cancer associated with improved survival: NRG Oncology/ Gynecologic Oncology Group study. Ann Oncol. 2016;27 (1):114-121. doi:10.1093/annonc/mdv500

6. Kohandel M, Sivaloganathan S, Oza A. Mathematical modeling of ovarian cancer treatments: sequencing of surgery and chemotherapy. $J$ Theor Biol. 2006;242(1):62-68. doi:10.1016/j.jtbi.2006.02.001

7. Kang KM, Hong KS, Noh GT, et al. Optimal time of initiating adjuvant chemotherapy after curative surgery in colorectal cancer patients. Ann Coloproctol. 2013;29(4):150-154. doi:10.3393/ ac.2013.29.4.150

8. Lohrisch C, Paltiel C, Gelmon K, et al. Impact on survival of time from definitive surgery to initiation of adjuvant chemotherapy for early-stage breast cancer. J Clin Oncol. 2006;24(30):4888-4894. doi:10.1200/JCO.2005.01.6089 
9. Gadducci A, Sartori E, Landoni F, et al. Relationship between time interval from primary surgery to the start of taxane- plus platinum-based chemotherapy and clinical outcome of patients with advanced epithelial ovarian cancer: results of a multicenter retrospective Italian study. J Clin Oncol. 2005;23(4):751-758. doi:10.1200/JCO.2005.03.065

10. Aletti GD, Long HJ, Podratz KC, Cliby WA. Is time to chemotherapy a determinant of prognosis in advanced-stage ovarian cancer? Gynecol Oncol. 2007;104(1):212-216. doi:10.1016/j.ygyno.2006.07.045

11. Zeng XT, Li S, Gong K, et al. [Evidence-based evaluation of recent clinical practice guidelines for the diagnosis and treatment of benign prostatic hyperplasia]. Zhonghua Yi Xue Za Zhi. 2017;97(22):1683-1687. doi:10.3760/cma.j.issn.0376-2491.2017.22.002. Chinese.

12. Rocher G, Gaillard T, Uzan C, et al. Does time-to-chemotherapy after primary complete macroscopic cytoreductive surgery influence prognosis for patients with epithelial ovarian cancer? A study of the FRANCOGYN group. J Clin Med. 2021;10(5):1058. doi:10.3390/ jcm10051058

13. Bookman MA, Okamoto A, Stuart G, et al. Harmonising clinical trials within the Gynecologic Cancer InterGroup: consensus and unmet needs from the Fifth Ovarian Cancer Consensus Conference. Ann Oncol. 2017;28(suppl_8):viii30-viii35. doi:10.1093/annonc/mdx449

14. Lydiksen L, Jensen-Fangel S, Blaakaer J. Is it possible to define an optimal time for chemotherapy after surgery for ovarian cancer? Gynecol Oncol. 2014;133(3):454 459. doi:10.1016/j.ygyno.2014.04.004

15. Wright J, Doan T, McBride R, Jacobson J, Hershman D. Variability in chemotherapy delivery for elderly women with advanced stage ovarian cancer and its impact on survival. Br J Cancer. 2008;98 (7):1197-1203. doi:10.1038/sj.bjc.6604298

16. Timmermans M, van der Aa MA, Lalisang RI, et al. Interval between debulking surgery and adjuvant chemotherapy is associated with overall survival in patients with advanced ovarian cancer. Gynecol Oncol. 2018;150(3):446-450. doi:10.1016/j.ygyno.2018.07.004

17. Seagle BL, Butler SK, Strohl AE, Nieves-Neira W, Shahabi S. Chemotherapy delay after primary debulking surgery for ovarian cancer. Gynecol Oncol. 2017;144(2):260-265. doi:10.1016/j. ygyno.2016.11.022

18. Selvin S. Two issues concerning the analysis of grouped data. Eur J Epidemiol. 1987;3(3):284-287. doi:10.1007/BF00149737

19. Altman DG. Categorizing continuous variables. Wiley StatsRef: Statistics Reference Online. 2014.

20. Manning-Geist BL, Hicks-Courant K, Gockley AA, et al. Moving beyond "complete surgical resection" and "optimal": is low-volume residual disease another option for primary debulking surgery? Gynecol Oncol. 2018;150(2):233-238. doi:10.1016/j.ygyno.2018.06.015
21. Hofstetter G, Concin N, Braicu I, et al. The time interval from surgery to start of chemotherapy significantly impacts prognosis in patients with advanced serous ovarian carcinoma - analysis of patient data in the prospective OVCAD study. Gynecol Oncol. 2013;131 (1):15-20. doi:10.1016/j.ygyno.2013.07.086

22. Wright JD, Herzog TJ, Neugut AI, et al. Effect of radical cytoreductive surgery on omission and delay of chemotherapy for advanced-stage ovarian cancer. Obstet Gynecol. 2012;120 (4):871-881. doi:10.1097/AOG.0b013e31826981de

23. Fotopoulou C, Jones BP, Savvatis K, et al. Maximal effort cytoreductive surgery for disseminated ovarian cancer in a UK setting: challenges and possibilities. Arch Gynecol Obstet. 2016;294 (3):607-614. doi:10.1007/s00404-016-4080-3

24. Flynn PM, Paul J, Cruickshank DJ. Does the interval from primary surgery to chemotherapy influence progression-free survival in ovarian cancer? Gynecol Oncol. 2002;86(3):354-357. doi:10.1006/ gyno.2002.6750

25. Usón PLJ, Bugano DD, França MS, et al. Does time-tochemotherapy impact the outcomes of resected ovarian cancer? Meta-analysis of randomized and observational data. Int J Gynecol Cancer. 2017;27(2):274-280. doi:10.1097/igc.0000000000000923

26. Mahner S, Eulenburg C, Staehle A, et al. Prognostic impact of the time interval between surgery and chemotherapy in advanced ovarian cancer: analysis of prospective randomised phase III trials. Eur $J$ Cancer. 2013;49(1):142-149. doi:10.1016/j.ejca.2012.07.023

27. Fisher B, Gunduz N, Saffer EA. Influence of the interval between primary tumor removal and chemotherapy on kinetics and growth of metastases. Cancer Res. 1983;43(4):1488-1492.

28. Schiffenbauer YS, Abramovitch R, Meir G, et al. Loss of ovarian function promotes angiogenesis in human ovarian carcinoma. Proc Natl Acad Sci U S A. 1997;94(24):13203-13208. doi:10.1073/ pnas.94.24.13203

29. Oza AM, Cook AD, Pfisterer J, et al. ICON7 trial investigators. Standard chemotherapy with or without bevacizumab for women with newly diagnosed ovarian cancer (ICON7): overall survival results of a Phase 3 randomised trial. Lancet Oncol. 2015;16 (8):928-936. doi:10.1016/S1470-2045(15)00086-8

30. Burger RA, Brady MF, Bookman MA, et al. Gynecologic Oncology Group. Incorporation of bevacizumab in the primary treatment of ovarian cancer. $N$ Engl $J$ Med. 2011;365(26):2473-2483. doi:10.1056/NEJMoa1104390

\section{Publish your work in this journal}

Cancer Management and Research is an international, peer-reviewed open access journal focusing on cancer research and the optimal use of preventative and integrated treatment interventions to achieve improved outcomes, enhanced survival and quality of life for the cancer patient.
The manuscript management system is completely online and includes a very quick and fair peer-review system, which is all easy to use. Visit http://www.dovepress.com/testimonials.php to read real quotes from published authors. 